\title{
A unified synthetic strategy to introduce heteroatoms via electrochemical functionalization of alkyl organoboron reagents
}

Su Yong Go ${ }^{1,3}$, Hyunho Chung ${ }^{1,3}$, Samuel Jaeho Shin ${ }^{1}$, Sohee An ${ }^{1}, J_{u}$ Hyun Youn ${ }^{1}$, Tae Yeong $\operatorname{Im}^{1}$, Ji Yong Kim ${ }^{1}$, Taek Dong Chung ${ }^{1,2,4}$ and Hong Geun Lee ${ }^{1,4}$

${ }^{1}$ Department of Chemistry, College of Natural Science, Seoul National University, 1 Gwanakro, Seoul 08826, Republic of Korea.

${ }^{2}$ Advanced Institutes of Convergence Technology, Suwon-si, Gyeonggi-do, 16229, Republic of Korea.

${ }^{3}$ These authors contributed equally: Su Yong Go and Hyunho Chung.

${ }^{4}$ These authors jointly supervised this work: Taek Dong Chung and Hong Geun Lee.

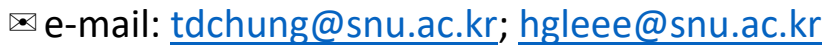

\begin{abstract}
Based on systematic electrochemical analyses, an integrated synthetic platform of $\mathrm{C}\left(\mathrm{sp}^{3}\right)$ based organoboron compounds was established for the introduction of heteroatoms. The electrochemically mediated bond-forming strategy was shown to be highly effective for the functionalization of $\mathrm{sp}^{3}$-hybridized carbon atoms with significant steric hindrance. Moreover, virtually all the nonmetallic heteroatoms could be utilized as reaction partners using one unified protocol. The observed reactivity stems from the two consecutive single-electron oxidations of the substrate, which eventually generates an extremely reactive carbocation as the key intermediate. The detailed reaction profile could be elucidated through multifaceted electrochemical studies and the examination of the reaction kinetics. Ultimately, a new dimension in the activation strategies for organoboron compounds was accomplished through the electrochemically driven reaction development.
\end{abstract}

\section{Main text}

The backbone structure of organic compounds comprises an array of carbon-based fragments, and the functional variety of the complied framework is embellished by the presence of polar heteroatoms that are usually attached to an $\mathrm{sp}^{3}$ - or $\mathrm{sp}^{2}$-hybridized carbon atom. The diversity given to the organic molecules by the placement of heteroatoms creates unique physical properties that allow the resulting organic chemical space to permeate a variety of disciplines in academic and industrial research (Fig. 1a). ${ }^{1-5}$ As such, the programmable introduction of a series of heteroatoms has been an enduring subject of investigation since the beginning of chemical synthesis.

Among various approaches, the chemically intuitive functionalization of an existing handle should serve as an optimal strategy for the general installation of heteroatoms (Fig. 1b). By conserving the hybridization state of the basal carbon, the structural reorganization of the precursor is minimized, thereby reducing the risks associated with the transformation. The handle-based introduction of a heteroatom on an $\mathrm{sp}^{2}$-hybridized carbon has allowed revolutionary advances owing to the emerging synthetic modalities that generate reactive species. $^{6-11}$ However, a great challenge resides in establishing an analogous mode of activation for $\mathrm{C}\left(\mathrm{sp}^{3}\right)$-based precursors. Mechanistic constraints embedded in the $\mathrm{C}\left(\mathrm{sp}^{3}\right)$ reaction center and increased steric congestion, which can encompass the $\mathrm{sp}^{3}$-hybridized carbon atom, make the introduction of heteroatoms particularly difficult. Consequently, many of the state-of-the-art methods for the construction of $\mathrm{C}\left(\mathrm{sp}^{3}\right)$-heteroatom bonds rely 
largely on traditional reactivities, such as facilitated displacement or in situ activation of a leaving group. ${ }^{12,13}$ Despite these difficulties, remarkable progress has recently been made toward disentangling numerous problems in this area. ${ }^{14-22}$ Notably, several strategies overcome the steric congestion of the reaction partners through the creation of highly reactive intermediates. ${ }^{17-21}$ However, even these advanced systems exhibit limitations imposed by the difficulties in installing the reactive handles and activating them in a chemoselective manner. Moreover, specialized reactivity for specific reactant-heteroatom combinations reduces the level of generality regarding the types of installable heteroatoms.

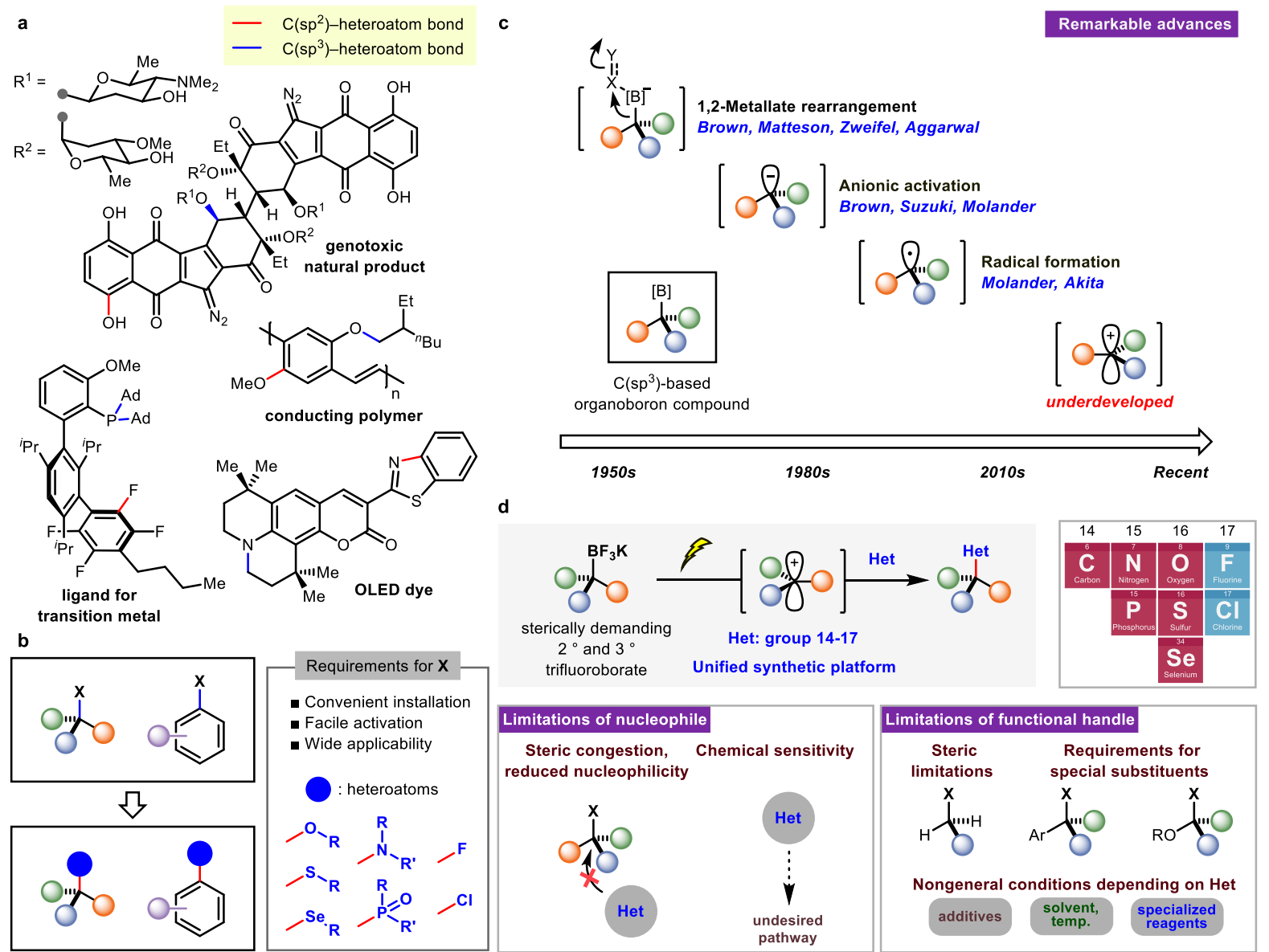

Figure 1. Introduction of heteroatoms into organic molecules and the utilization of the C(sp $\left.{ }^{3}\right)-B$ bond as a tool. a, Functional organic molecules containing $\mathrm{C}\left(\mathrm{sp}^{2}\right)$ - or $\mathrm{C}\left(\mathrm{sp}^{3}\right)$-heteroatom bonds. b, Introduction of heteroatoms into $\mathrm{sp}^{3}$ - or $\mathrm{sp}^{2}$-hybridized carbon atom using a functional handle. $\mathbf{c}$, Activation strategies of organoboron compounds. d, Cationic activation of organoboron compounds via electrochemical oxidation. Het, heteroatom.

One of the optimal functional handles that meets the ideality/universality requirements for heteroatom introduction at an $\mathrm{sp}^{3}$-hybridized carbon is the carbon-boron bond. In terms of the ease of installation, the carbon-metal bond is one of the most convenient functional groups that can be introduced to a variety of positions on organic molecules with the help of recently developed borylation techniques. ${ }^{23}$ The classical activation strategies of the $\mathrm{C}\left(\mathrm{sp}^{3}\right)$-based organoboron reagents have evolved on the basis of the 1,2-rearrangement of their ate-complex form (Fig. 1c). ${ }^{24-27}$ Overtime, the utility of the compound has been extended to the carbanionic nature towards electrophilic reaction counterparts. ${ }^{28-30}$ Most recently, a single-electron-transfer-based approach has widened the 
reactivity scope of the handle by providing a radical intermediate that can mediate numerous useful transformations. ${ }^{31-34}$ However, a functionalization approach to form a carbon-centered cation from $\mathrm{C}\left(\mathrm{sp}^{3}\right)$-based organoboron compounds, despite their suitability for interacting with polar heteroatom-based reaction partners, is extremely rare in the synthetic context. Indeed, basic reactivity has been noted, and organoboron compounds could be utilized to generate reactive carbocation intermediates under oxidative conditions in both a chemical and electrochemical manner. ${ }^{35-37}$ However, attempts to explore the synthetic feasibility of this strategy are still in their infancy because of the limitations enforced by the reaction parameters, such as the unfavorable stoichiometry of the reaction partners and/or harsh conditions. In addition, there is no systematic assessment of the system to scrutinize the formation of the reactive intermediate. We envisioned that a controlled carbocationic activation of the $\mathrm{C}\left(\mathrm{sp}^{3}\right)$-based organometallic reagent could offer a universally applicable method for the introduction of a wide range of heteroatoms. Of the numerous oxidation strategies available, the electrochemically mediated process should deliver the required reactive intermediate in the most efficient and regulated fashion. ${ }^{38-40}$ Particularly inspiring is the fact that the carbocation is a key intermediate in other notable approaches to overcoming important synthetic hurdles, especially steric congestion. ${ }^{17-21}$

Herein, we demonstrate the synthetic capability of organoboron reagents to construct $\mathrm{C}\left(\mathrm{sp}^{3}\right)$-heteroatom bonds in a preparatively ideal manner (Fig. 1d). By generating highly reactive carbocations via programmable and energy-efficient anodic oxidation in an electrochemical reaction system, the desired reactivity was achieved. A multitude of heteroatoms, even with unfavorable properties such as steric congestion, reduced nucleophilicity, redox vulnerability, or chemical sensitivity, could be introduced under a single unified activation platform. During the course, the conventional limitations of the counterpart, including steric restrictions, requirements for special substituents, or customization of the reaction conditions for each reactant, were also addressed. Eventually, a challenge associated with the universality of the method, which have stymied research efforts in other disciplines for decades, has been settled in an extremely expeditious fashion.

At the outset, a rational approach based on the voltametrically confirmed oxidation profile of the organotrifluoroborate was utilized (Fig. 2a). The initial electrochemical assessments of hindered trifluoroborate substrates $\mathbf{A}$ and $\mathbf{B}$ were made using cyclic voltammetry under an inert atmosphere. This resulted in the identification of two distinct peaks representing anodic oxidations. Further analyses with differential pulse voltammetry and square wave voltammetry demonstrated analogous results with an increased level of distinction (See the supplementary information for details). ${ }^{41-43}$ Thus, the collected evidence clearly shows that two successive oxidation events of organoboron compounds are taking place. A more quantitative evaluation of the event was performed using a thin-layer electroanalysis microchip (TEAM) (Fig. 2b). ${ }^{44}$ A representative model substrate, trifluoroborate $\mathbf{C}$, underwent the first oxidation event at $1.1 \mathrm{~V}$ with the number of electrons transferred per molecule, $n$, being equal to unity. In contrast, $\mathbf{C}$ at $1.5 \mathrm{~V}$ showed the $n$ value virtually converging to 2 , suggesting the involvement of an additional oxidation under the given potential. Based on these electrochemical analyses, we hypothesized that the two successive electrochemical oxidation processes would generate the postulated carbocation intermediate to enable the desired bond formation. 

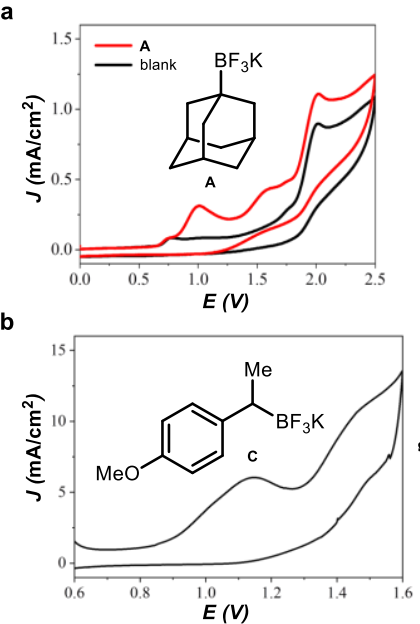
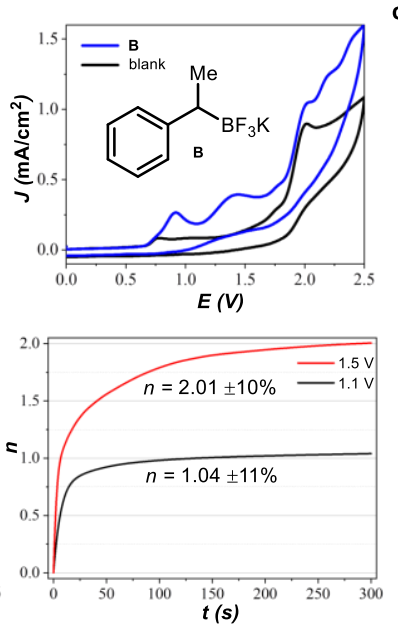

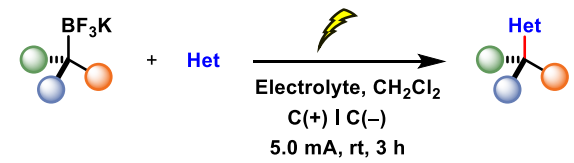

Highlighted feature

- Straightforward setup

- $\mathrm{CH}_{2} \mathrm{Cl}_{2}$ as electron-sink

- High Faradaic efficiency
Dispensable elements

- Acid or base

- External oxidant or reductant

- Drying agent

- Specialized electrode

Figure 2. Identification of reaction conditions on the basis of electrochemical analyses. a, Cyclic voltammograms of alkyl trifluoroborate $\mathbf{A}(1.0 \mathrm{mM})$ and $\mathbf{B}(1.0 \mathrm{mM})$ in $\mathrm{CH}_{2} \mathrm{Cl}_{2}$ with ${ }^{n} \mathrm{Bu}_{4} \mathrm{NPF}_{6}(0.1 \mathrm{M})$ supporting electrolyte compared to that of blank (electrolyte solution) on glassy carbon electrode with the scan rate of $100 \mathrm{mV} / \mathrm{s}$. b, Cyclic voltammogram $(10 \mathrm{mV} / \mathrm{s})$ and $n$ - $t$ plots $\left(E_{\text {app }}=1.1\right.$ and $\left.1.5 \mathrm{~V}\right)$ of $\mathbf{C}(1.0 \mathrm{mM})$ in $\mathrm{CH}_{2} \mathrm{Cl}_{2}$ with ${ }^{n} \mathrm{Bu}_{4} \mathrm{NPF}_{6}(0.1 \mathrm{M})$ supporting electrolyte obtained by TEAM. c, Optimized reaction parameters. ${ }^{n} \mathrm{Bu}_{4} \mathrm{NPF}_{6}$, tetra$n$-butylammonium hexafluorophosphate; $\mathrm{C}(+) \mid \mathrm{C}(-)$ represents undivided cell with graphite electrodes.

Based on the electrochemical analyses, a general strategy for the functionalization of $\mathrm{C}\left(\mathrm{sp}^{3}\right)-\mathrm{B}$ bonds was established, which ultimately permitted the introduction of a range of heteroatoms (Fig. 2c). Some notable conclusions drawn from the multidimensional reaction parameter evaluation include the following: (a) external additives, such as oxidants, bases, or dehydrating agents were demonstrated to be non-essential; (b) an inexpensive carbonbased electrode, the graphite electrode, furnished the desired coupling product with the greatest efficiency; and (c) the use of dichloromethane as the reaction solvent, which also acts as an electron sink, ${ }^{18,45}$ elicited the best performance (See the supplementary information for details). On the electrochemical side, the Faradaic efficiency of the system was typically in the $40-80 \%$ range with a maximum of $93 \%$; hence, the transformation is highly energy efficient. In addition, a current of $5.0 \mathrm{~mA}$, which resulted in a 5.0-6.0 V initial cell voltage between the anode and cathode, was demonstrated to be optimal, although deviation from $5.0 \mathrm{~mA}$ did not significantly affect the outcome. In summary, the formation of $\mathrm{C}\left(\mathrm{sp}^{3}\right)$-heteroatom bonds under constant current in a simple undivided cell was generally achieved within 3 hours at room temperature. 


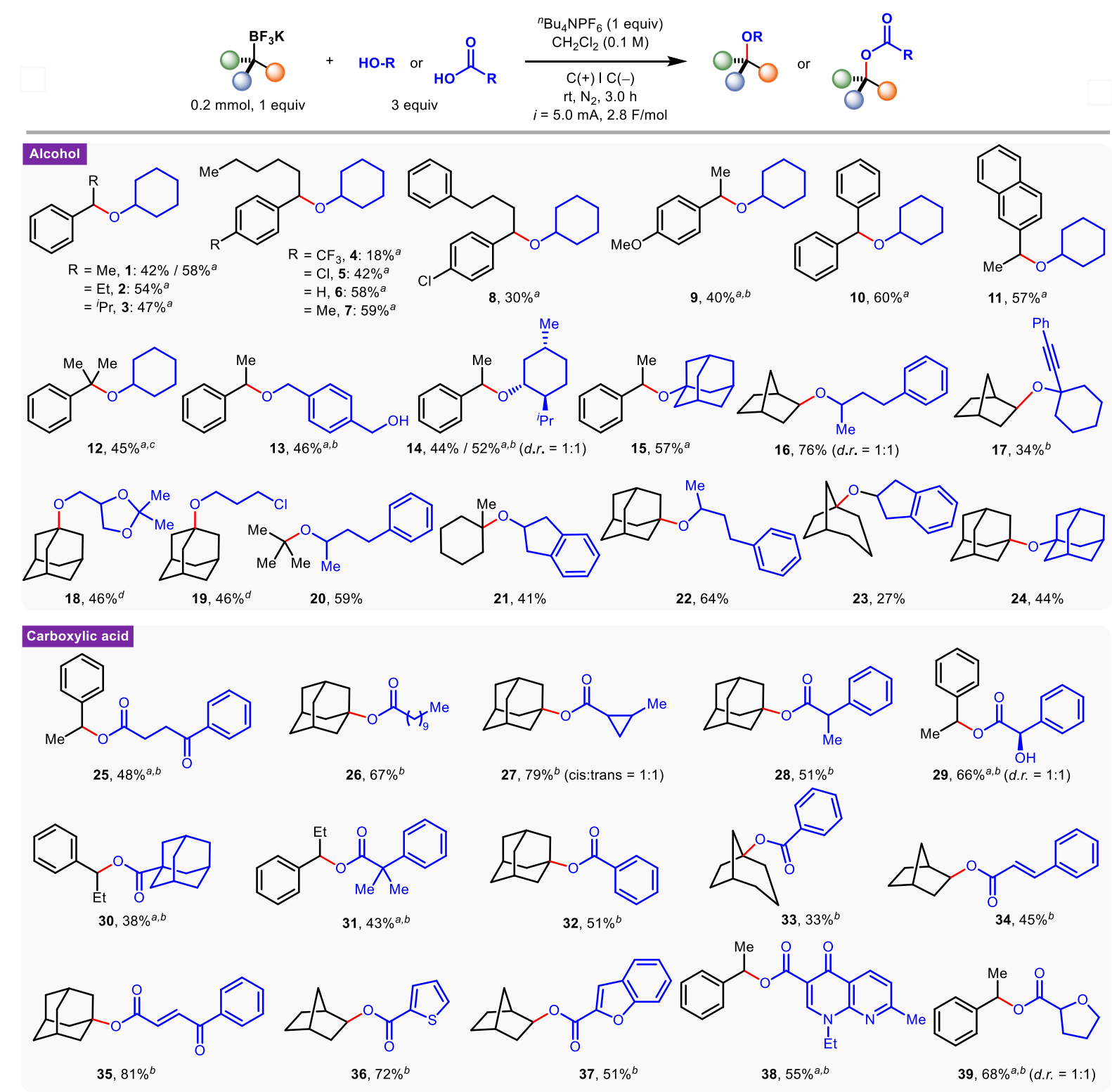

Table 1. Synthesis of sterically congested ethers and esters by the introduction of oxygen nucleophiles. ${ }^{a}$ ${ }^{n} \mathrm{Bu}_{4} \mathrm{NClO}_{4}$ (1 equiv) was used as an electrolyte instead of ${ }^{n} \mathrm{Bu}_{4} \mathrm{NPF}_{6}$ (1 equiv). ${ }^{b}$ The reaction was run for $2.0 \mathrm{~h}$ instead of $3.0 \mathrm{~h}$. 'The reaction was run for $1.5 \mathrm{~h}$ instead of $3 \mathrm{~h}$. ${ }^{d} 6$ equivalent of the nucleophile was used. ${ }^{n} \mathrm{Bu}_{4} \mathrm{NClO}_{4}$, tetra- $n$-butylammonium perchlorate; rt, room temperature; $d . r$., diastereomeric ratio; $i$ represents constant current.

Initially, the strategy was applied to the construction of a $\mathrm{C}\left(\mathrm{sp}^{3}\right)-\mathrm{O}$ bond with an alcohol or a carboxylic acid as a nucleophile (Table 1). In the presence of an alcohol nucleophile, sterically encumbered benzylic trifluoroborate substrates underwent smooth ether formation (1-15). Secondary benzylic substrates furnished the corresponding ethers with cyclohexanol regardless of the increased steric demand around the reaction center (13). On the other hand, variations in the electronic nature of the substrates significantly affected the reaction outcomes (4-7). As the arene ring became richer in electron density, the efficiency of product formation increased distinctly. The observed trend indicates the involvement of a reactive intermediate with carbocationic character. Other types of benzylic systems containing multiple arene rings $(\mathbf{8}, \mathbf{1 0})$, an extended $\pi$-system $(\mathbf{1 1})$, or a tertiary reaction center (12) also underwent facile alkoxylation under the given conditions. In the 
case of the alcohol nucleophiles, oxidatively labile 1,4-benzenedimethanol (13) and sterically demanding secondary (14) or tertiary (15) carbinol provided the coupling products with high efficiency. Aliphatic secondary and tertiary trifluoroborates were also viable substrates for the transformation (16-24). Sterically congested $\alpha$-tertiary dialkyl ether products could be obtained in a highly straightforward manner using a variety of alcohol substrates (17-24). Importantly, alcohols possessing sensitive functional groups such as an alkyne, an acetal, or a chloride underwent the desired transformation without losing their structural integrity (17-19).

Subsequently, the reactivity scope of weaker oxygen-based nucleophiles, carboxylic acids, was assessed to afford hindered esters. Various aliphatic and aromatic carboxylic acids could conveniently be introduced to the $\mathrm{C}\left(\mathrm{sp}^{3}\right)$-center of the organoboron reagent to furnish sterically congested ester products (25-39). Notably, carboxylic acids that are vulnerable to oxidation under other electrochemical oxidation conditions could be utilized as reaction partners without competitive generation of reactive species $(\mathbf{2 5 - 3 1}, 39) .{ }^{46}$ Moreover, highly reactive $\alpha, \beta$-unsaturated carbonyl systems (34-35) and biomedically important heterocycles (36-39) were tolerated. Remarkably, in the case of mandelic acid, which contains a hydroxyl group, the generated alkyl carbocation selectively reacted with the carboxylic acid moiety (29). 

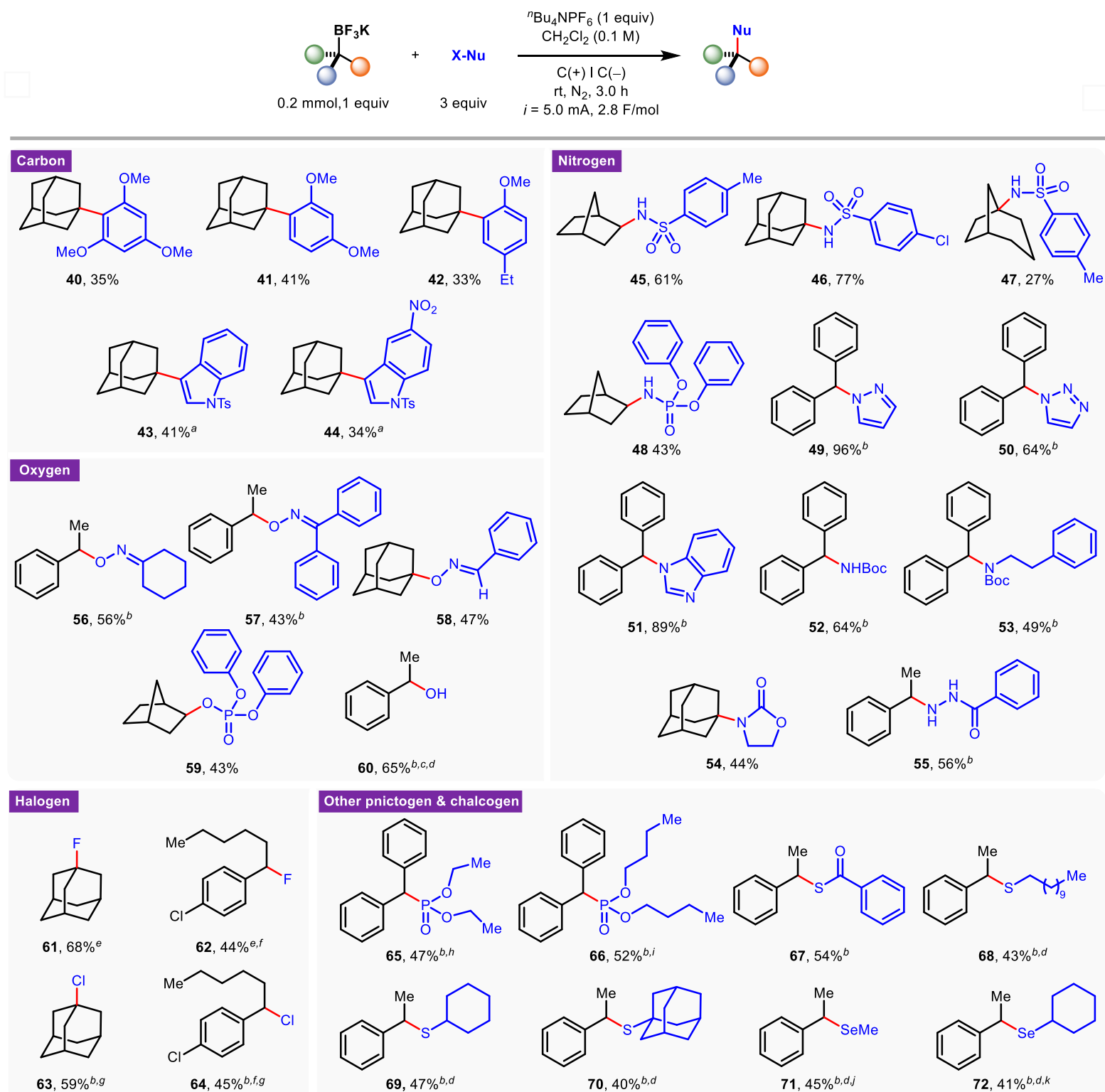

Table 2. Introduction of other heteroatoms into an $\mathbf{s p}^{3}$-hybridized carbon atom. ${ }^{a}$ The reaction was set up with alkyl trifluoroborate $\left(0.1 \mathrm{mmol}\right.$ ), nucleophile (2 equiv), and ${ }^{n} \mathrm{Bu}_{4} \mathrm{NPF}_{6}$ (2 equiv) in $\mathrm{CH}_{2} \mathrm{Cl}_{2}(0.05 \mathrm{M})$ with 3.0 $\mathrm{mA}$ of electric current. ${ }^{b} \mathrm{Bu}_{4} \mathrm{NClO}_{4}$ (1 equiv) was used as an electrolyte instead of ${ }^{n} \mathrm{Bu}_{4} \mathrm{NPF}_{6}$ (1 equiv). ${ }^{c} \mathrm{H}_{2} \mathrm{O}$ (0.1 $\mathrm{mL}$ ) was used as a nucleophile. ${ }^{d}$ The reaction was run for $1.5 \mathrm{~h}$ instead of $3.0 \mathrm{~h}$. ${ }^{e} \mathrm{KHF}_{2}$ (2 equiv) was used as a nucleophile with 18-crown-6 (2 equiv). ${ }^{f}$ The reaction was run for $2.0 \mathrm{~h}$ instead of $3.0 \mathrm{~h} .{ }^{g} \mathrm{KCl}(2$ equiv) was used as a nucleophile with 18-crown-6 (2 equiv). ${ }^{h}$ Triethyl phosphite (3 equiv) was used as a source of heteroatom. 'Tributhyl phosphite (3 equiv) was used as a source of heteroatom. ${ }^{j 1,2-D i m e t h y l d i s e l a n e ~(3 ~ e q u i v) ~ w a s ~ u s e d ~ a s ~}$ a nucleophile. ${ }^{k} 1,2-$-Dicyclohexyldiselane (3 equiv) was used as a nucleophile. Ts, tosyl; Boc, tertbutyloxycarbonyl)

The recognized reactivity of organotrifluoroborate was exploited to form other types of sterically challenged chemical bonds at $\mathrm{sp}^{3}$-hybridized carbons (Table 2). Essentially, the electrochemically generated reactive intermediate could form a covalent bond with virtually all nonmetallic elements in the second and third rows. A variety of electron-rich arenes participated in the formation of $\mathrm{C}\left(\mathrm{sp}^{3}\right)-\mathrm{C}\left(\mathrm{sp}^{2}\right)$ bonds in a highly programmable manner with regard to regioselectivity (40-42). Interestingly, bioactive indole derivatives with attenuated electron densities can react with a sterically demanding $C\left(\mathrm{sp}^{3}\right)$ building block $(\mathbf{4 3}, \mathbf{4 4})$. Furthermore, the regioselective formation of C3-alkylation products of indoles suggests that 
a carbocation intermediate is responsible for bond formation rather than a radical species. ${ }^{47}$ Several nitrogen-based nucleophiles were also found to be viable reaction partners for this method (45-55). Hindered $\mathrm{C}\left(\mathrm{sp}^{3}\right)-\mathrm{N}$ bonds of sulfonamides and a phosphoramidate could be formed efficiently (45-48). An array of pharmaceutically important azoles could participate in $\mathrm{C}\left(\mathrm{sp}^{3}\right)-\mathrm{N}$ bond formation in synthetically useful yields (49-51) ${ }^{48}$ Of importance, alkylation of carbamates was also feasible for furnishing highly substituted carbamate products (5254). In principle, access to complex amines should be possible via simple deprotection of the tert-butyloxycarbonyl protecting group from the carbamate products $(\mathbf{5 2}, \mathbf{5 3})$. Moreover, a redox-sensitive acylhydrazide was selectively alkylated on the terminal nitrogen, demonstrating the mildness of the protocol (55). This strategy has also been applied to more complex oxygen-based nucleophiles that are not alcohols or carboxylic acids. Medicinally important oxime scaffolds could be expanded by the facile introduction of bulky alkyl groups on the oxygen (56-58). Aliphatic/aromatic ketoximes and an aldoxime were equally competent in terms of $\mathrm{C}\left(\mathrm{sp}^{3}\right)-\mathrm{O}$ bond construction. It is anticipated that the preparation of a complex oxime structure should allow for the synthesis of related alkoxyamines with academic and industrial importance via straightforward transformations. In addition, a mixed phosphate triester could be formed by the reaction of an extremely weak oxygen nucleophile (59). The conversion of a hindered borate to the corresponding alcohol without using any chemical oxidants was also shown to be viable (60). Finally, fluorinated or chlorinated products could be accessed by utilizing potassium hydrogen difluorides $\left(\mathrm{KHF}_{2}\right)$ or potassium chloride $(\mathrm{KCl})$ as halogen sources (61-64).

Furthermore, the established methodology was applicable to bond formation reactions with pnictogen and chalcogen elements in the third and fourth rows. Trialkyl phosphite-based reaction partners participated in a dealkylative $\mathrm{C}\left(\mathrm{sp}^{3}\right)-\mathrm{P}$ bond construction reaction to yield hindered phosphonated products (65-66). Sulfur-containing compounds, such as a thioacid or thiols are also competent reaction components that furnish a variety of organosulfur compounds (67-70). Finally, an extension of this strategy could be exploited to synthesize oxidatively sensitive selenides in a straightforward manner (71-72). Of note, the sources of Group 16 nucleophiles are in their native form, which is inherently the most stable and therefore the easiest to manipulate. Overall, the introduction of numerous heteroatoms to the carbon backbone structure via only one unified protocol was realized to create new heteroatom-containing scaffolds. 


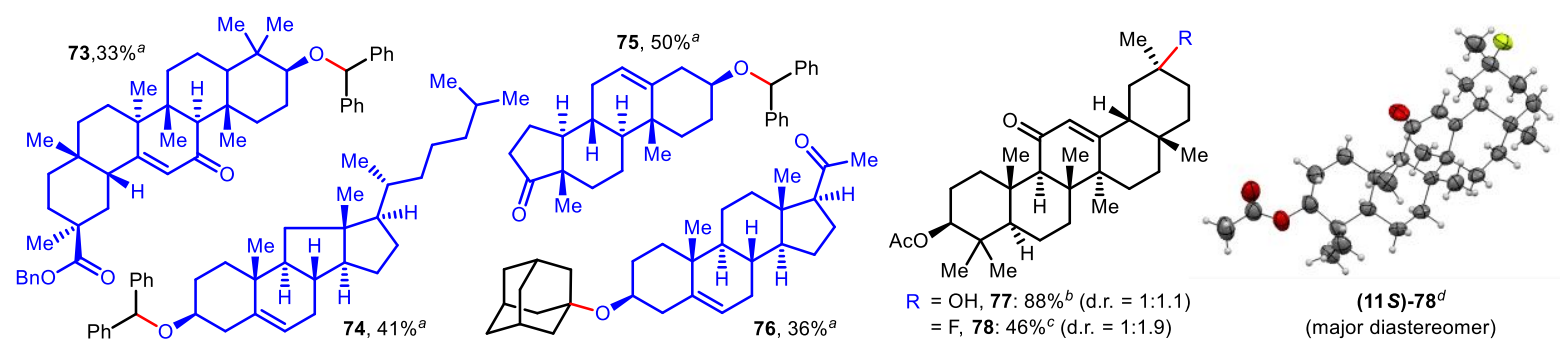

b

C

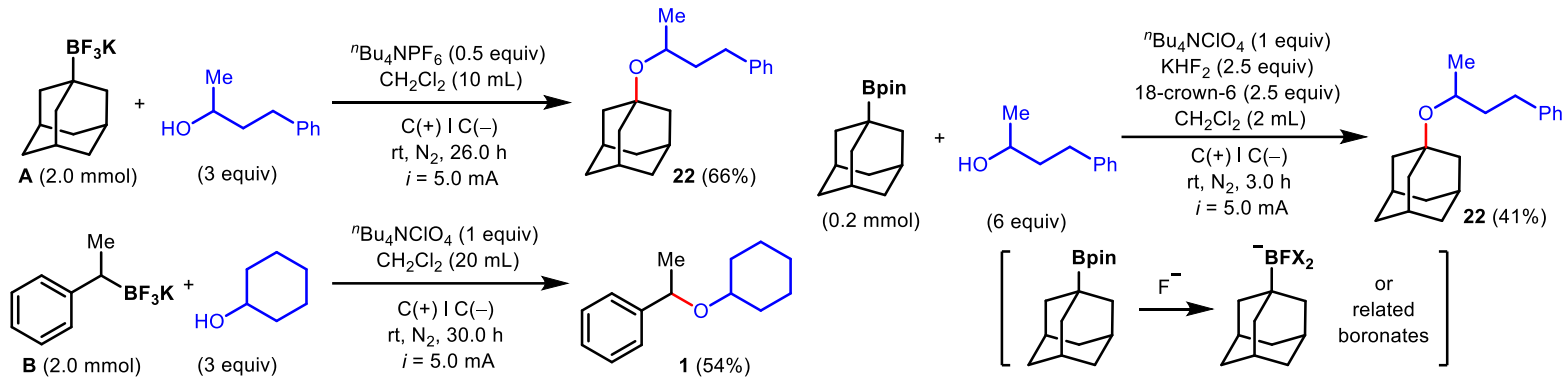

Figure 3. Synthetic applications of the developed strategy. a, Synthesis of complex steroid derivatives. ${ }^{a}$ The reaction was set up with alkyl trifluoroborate $(0.2 \mathrm{mmol})$, alcohol nucleophile (2 equiv), and ${ }^{n} \mathrm{Bu}_{4} \mathrm{NClO}_{4}(1$ equiv) in $\mathrm{CH}_{2} \mathrm{Cl}_{2}(2 \mathrm{~mL})$ with $10.0 \mathrm{~mA}$ of electric current. The reaction was run for $2.0 \mathrm{~h}$ at $\mathrm{rt}$. ${ }^{b}$ The reaction was set up with alkyl trifluoroborate $(0.2 \mathrm{mmol}), \mathrm{H}_{2} \mathrm{O}(0.1 \mathrm{~mL})$, and ${ }^{n} \mathrm{Bu}_{4} \mathrm{NPF}_{6}$ (1 equiv) in $\mathrm{CH}_{2} \mathrm{Cl}_{2}(2 \mathrm{~mL})$ with $5.0 \mathrm{~mA}$ of electric current. The reaction was run for $3.0 \mathrm{~h}$ at $\mathrm{rt}$. 'The reaction was set up with alkyl trifluoroborate $(0.2$ mmol), $\mathrm{KHF}_{2}$ (2 equiv), 18-crown-6 (2 equiv), and ${ }^{n} \mathrm{Bu}_{4} \mathrm{NPF}_{6}$ (1 equiv) in $\mathrm{CH}_{2} \mathrm{Cl}_{2}$ (2 mL) with $5.0 \mathrm{~mA}$ of electric current. The reaction was run for $2.0 \mathrm{~h}$ at $\mathrm{rt}$. ${ }^{d}$ See Supplementary Information for the X-ray crystallographic analysis of (11S)-78. b, Larger scale reaction. c, In situ generation of the reactive precursor. pin, pinacolato.

The synthetic competence of the developed methodology was further demonstrated by the preparation of complex molecules based on a steroidal backbone structure (Fig. 3a). The hydroxyl groups of complex steroid natural products or their derivatives have successfully participated in this bond formation, demonstrating the robustness of the protocol (73-76). Moreover, a complex organoboron compound originating from enoxolone could be successfully employed as a coupling partner to deliver valuable products with an added heteroatom substituent $(\mathbf{7 7}, \mathbf{7 8})$. Additionally, the robustness and practicality of the method were evaluated based on a preparatory-scale reaction setup (Fig. $3 b$ ). When the reaction scale was increased 10 fold, the reaction yield remained virtually unchanged, although a longer reaction time was required. Lastly, to facilitate a more practical operation of the strategy, a more easily accessible organoboron substrate was directly used as a reaction partner (Fig. 3c). The incorporation of a fluoride source into the reaction system was expected to induce in situ generation of fluoroboronate derivatives, thereby simplifying the overall process. Indeed, the step-economical pathway starting from alkyl pinacolboronate, one of the immediate precursors of trifluoroborate, successfully provided the desired product with comparable efficiency. Overall, the strength of the protocol as a synthetic tool has been clearly demonstrated in multiple ways. 

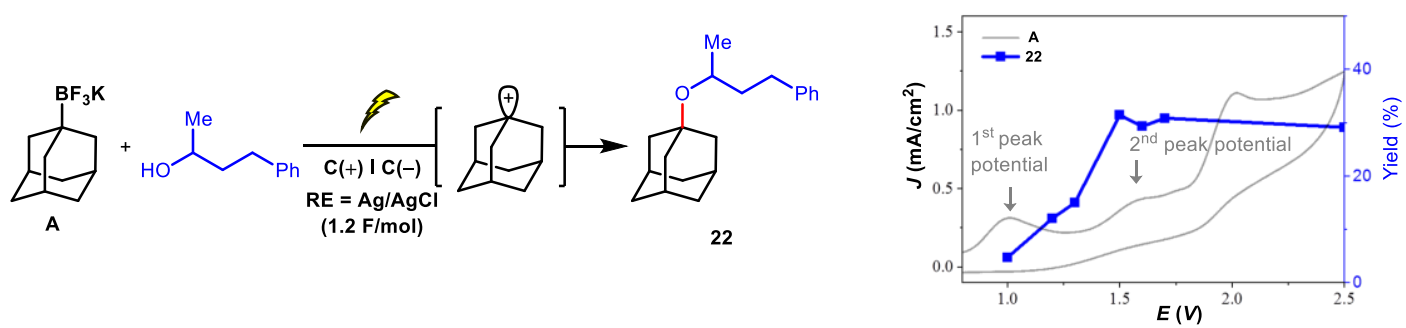

b
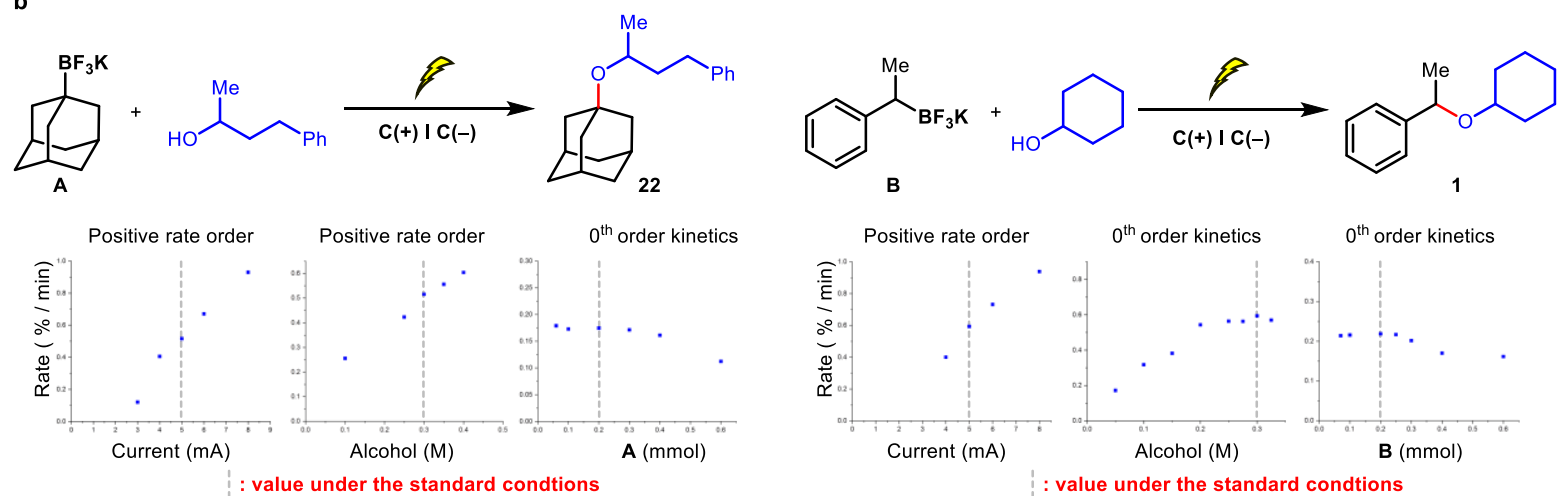

Figure 4. Mechanistic studies of the developed synthetic platform. a, Constant potential electrolysis. ${ }^{a}$ The reaction was set up with alkyl trifluoroborate $\mathbf{A}\left(0.1 \mathrm{mmol}\right.$ ), alcohol (18 equiv), and ${ }^{n} \mathrm{Bu}_{4} \mathrm{NPF}_{6}\left(6\right.$ equiv) in $\mathrm{CH}_{2} \mathrm{Cl}_{2}$ $(6 \mathrm{~mL})$. The three-electrode system was used with graphites as the working and counter electrode, and $\mathrm{Ag} / \mathrm{AgCl}$ wire in $3.0 \mathrm{M} \mathrm{NaCl}$ as the reference electrode. Each data point at different potentials was obtained by running the reaction with a fixed total charge, $1.2 \mathrm{~F} / \mathrm{mol}$, that had passed through the circuit. The reaction yield was determined by gas chromatography using mesitylene as an internal standard. RE, reference electrode. b, Kinetic profiles. standard conditions: $\mathbf{A}(0.2 \mathrm{mmol})$, alcohol (3 equiv), ${ }^{n} \mathrm{Bu}_{4} \mathrm{NPF}_{6}$ (1 equiv), $\mathrm{CH}_{2} \mathrm{Cl}_{2}(2$ $\mathrm{mL}$ ), $i=5.0 \mathrm{~mA}$ (left). standard conditions: $\mathbf{B}\left(0.2 \mathrm{mmol}\right.$ ), alcohol (3 equiv), ${ }^{n} \mathrm{Bu}_{4} \mathrm{NClO}_{4}$ (1 equiv), $\mathrm{CH}_{2} \mathrm{Cl}_{2}(2 \mathrm{~mL}), i$ $=5.0 \mathrm{~mA}$ (right). The reaction rate was determined by gas chromatography using $n$-octane as an internal standard. The dotted vertical line designates the value of the corresponding physical property under the standard conditions.

Additional mechanistic information was collected to elucidate the detailed reaction pathways. The results of electrolysis experiments in a divided cell confirmed that product formation occurs exclusively in the anode chamber, suggesting that anodic oxidation is responsible for the observed reactivity (See the supplementary information for details). Furthermore, a 1,2-migration of the $\mathrm{C}\left(\mathrm{sp}^{3}\right)$-based reaction center could be encountered in agreement with the participation of a carbocation intermediate, a species that commonly rearranges to form a more stable isomer (See the supplementary information for details). A more detailed mechanistic scenario could be deduced from a constant potential electrolysis experiment using the reaction of adamantyl trifluoroborate and 4-phenyl-2-butanol as a model system (Fig. 4a). With the amount of total charge transfer remained unchanged, the reaction outcome was monitored by varying the applied potential. When the applied potential increased to $1.5 \mathrm{~V}$, a potential responsible for the second oxidation of the trifluoroborate substrate (Fig. 2a), an abrupt increase in the product yield was observed. Simultaneously, a gradual decrease in the yields of the side products, such as the protodeborylation and fluorination products, was detected. Therefore, it is concluded that the formation of carbocations will not be sufficiently effective until the reaction potential reaches a critical threshold, at which point the desired pathway prevails.

Finally, the kinetic profile of the system is also consistent with the described scenario. The model reaction to form the alkyl ether product $\mathbf{2 2}$ exhibited a positive rate order with respect to the applied current and the concentration of the alcohol nucleophile (Fig. 4b). In 
contrast, variation in the trifluoroborate concentration did not significantly influence the reaction rate, although an extremely high concentration induced a negative disturbance in the system. The apparent $S_{N} 2$-like reaction kinetics in terms of the concentration of anodically generated carbocation and the nucleophile concentration suggest the involvement of a borderline mechanism, in which carbocation generation may not be significantly slower during the $\mathrm{C}\left(\mathrm{sp}^{3}\right)-\mathrm{O}$ bond formation event. In fact, the rate of carbocation generation is highly dependent on the identity of the trifluoroborate substrate. The reaction rate profile of an alternative system to generate product $\mathbf{1}$ demonstrates the involvement of the rate-limiting oxidation of alkyl trifluoroborate salt on the anode to generate a carbocation species. Collectively, the efficiency of the designed $\mathrm{C}\left(\mathrm{sp}^{3}\right)_{-}$ heteroatom bond formation is a function of both the electrochemical activation of the organoboron substrate and the nucleophilicity of the heteroatom component. ${ }^{49}$

In summary, organoboron compounds have been evaluated from a synthetic perspective to enable the development of a universal $\mathrm{C}\left(\mathrm{sp}^{3}\right)$-heteroatom bond construction strategy conferring an unprecedented level of utility. The desired reactivity is derived from the electrochemically-driven two consecutive single-electron oxidations of the organometallic precursor, which generates an intermediate with elevated reactivity, a carbocation. Consequently, the strategy has allowed the development of a general synthetic platform whose effectiveness is not restricted by the nature or identity of the heteroatom reaction component. This method is highly suitable for the formation of $\mathrm{C}\left(\mathrm{sp}^{3}\right)$-heteroatom bonds at a sterically demanding reaction center and is applicable to the synthesis of complex molecules. Mechanistically, both the electrochemical generation of the carbocation intermediate and the heteroatom-carbocation combination are important factors that govern the overall efficiency. It is anticipated that the general synthetic platform reported herein will offer a variety of opportunities for the construction of $\mathrm{C}\left(\mathrm{sp}^{3}\right)$-heteroatom bonds in numerous settings. Additionally, the systematic electrochemical assessments demonstrated herein should provide guidance for other types of electrochemical reactions in the course of establishing the corresponding design principle.

\section{References}

1 Hartwig, J. F. Carbon-heteroatom bond formation catalysed by organometallic complexes. Nature 455, 314-322 (2008).

2 Herzon, S. B. The mechanism of action of (-)-Lomaiviticin A. Acc. Chem. Res. 50, 2577-2588 (2017).

3 Sather, A. C. et al. A fluorinated ligand enables room-temperature and regioselective Pd-catalyzed fluorination of aryl triflates and bromides. J. Am. Chem. Soc. 137, 13433-13438 (2015).

4 Chen, C. H. \& Tang, C. W. Efficient green organic light-emitting diodes with stericly hindered coumarin dopants. Appl. Phys. Lett. 79, 3711-3713 (2001).

5 Martin, S. J., Bradley, D. D. C., Lane, P. A., Mellor, H. \& Burn, P. L. Linear and nonlinear optical properties of the conjugated polymers PPV and MEH-PPV. Phys. Rev. B 59, 15133-15142 (1999).

6 Ruiz-Castillo, P. \& Buchwald, S. L. Applications of palladium-catalyzed C-N crosscoupling reactions. Chem. Rev. 116, 12564-12649 (2016).

7 Chen, J.-Q., Li, J.-H. \& Dong, Z.-B. A review on the latest progress of Chan-Lam coupling reaction. Adv. Synth. Catal. 362, 3311-3331 (2020). 
Barcellos, A. M. et al. Organoboron compounds as versatile reagents in the transition metal-catalyzed C-S, C-Se and C-Te bond formation. Coord. Chem. Rev. 442, 214012 (2021).

9 Cavedon, C., Seeberger, P. H. \& Pieber, B. Photochemical strategies for carbonheteroatom bond formation. Eur. J. Org. Chem. 2020, 1379-1392 (2020).

10 Zhu, C., Yue, H., Jia, J. \& Rueping, M. Nickel-catalyzed C-heteroatom cross-coupling reactions under mild conditions via facilitated reductive elimination. Angew. Chem. Int. Ed. 60, 17810-17831 (2021).

11 Walker, B. R., Manabe, S., Brusoe, A. T. \& Sevov, C. S. Mediator-enabled electrocatalysis with ligandless copper for anaerobic Chan-Lam coupling reactions. J. Am. Chem. Soc. 143, 6257-6265 (2021).

12 Liu, L., Tang, Y., Wang, K., Huang, T. \& Chen, T. Transition-metal-free and basepromoted carbon-heteroatom bond formation via $\mathrm{C}-\mathrm{N}$ cleavage of benzyl ammonium salts. J. Org. Chem. 86, 4159-4170 (2021).

13 Fletcher, S. The Mitsunobu reaction in the $21^{\text {st }}$ century. Org. Chem. Front. 2, 739-752 (2015).

14 Mao, R., Balon, J. \& Hu, X. Decarboxylative $\mathrm{C}\left(\mathrm{sp}^{3}\right)-\mathrm{O}$ cross-coupling. Angew. Chem. Int. Ed. 57, 13624-13628 (2018).

15 Mao, R., Frey, A., Balon, J. \& Hu, X. Decarboxylative $\mathrm{C}\left(\mathrm{sp}^{3}\right)-\mathrm{N}$ cross-coupling via synergetic photoredox and copper catalysis. Nat. Catal. 1, 120-126 (2018).

16 Liang, Y., Zhang, X. \& MacMillan, D. W. C. Decarboxylative $\mathrm{sp}^{3} \mathrm{C}-\mathrm{N}$ coupling via dual copper and photoredox catalysis. Nature 559, 83-88 (2018).

$17 \mathrm{Hu}, \mathrm{X}$., Zhang, G., Bu, F., Nie, L. \& Lei, A. Electrochemical-oxidation-induced siteselective intramolecular $\mathrm{C}\left(\mathrm{sp}^{3}\right)-\mathrm{H}$ amination. ACS Catal. 8, 9370-9375 (2018).

18 Xiang, J. et al. Hindered dialkyl ether synthesis with electrogenerated carbocations. Nature 573, 398-402 (2019).

19 Shibutani, S. et al. Organophotoredox-catalyzed decarboxylative $\mathrm{C}\left(\mathrm{sp}^{3}\right)-0$ bond formation. J. Am. Chem. Soc. 142, 1211-1216 (2020).

20 Wang, $\mathrm{H}$. et al. Electrochemical oxidation-induced etherification via $\mathrm{C}\left(\mathrm{sp}^{3}\right)-\mathrm{H} / \mathrm{O}-\mathrm{H}$ cross-coupling. Sci. Adv. 6, eaaz0590 (2020).

21 Lee, B. J., DeGlopper, K. S. \& Yoon, T. P. Site-selective alkoxylation of benzylic C-H bonds by photoredox catalysis. Angew. Chem. Int. Ed 59, 197-202 (2020).

22 Oliva, M., Coppola, G. A., Van der Eycken, E. V. \& Sharma, U. K. Photochemical and electrochemical strategies towards benzylic $\mathrm{C}-\mathrm{H}$ functionalization: a recent update. Adv. Synth. Catal. 363, 1810-1834 (2021).

23 Wang, M. \& Shi, Z. Methodologies and strategies for selective borylation of C-het and C-C bonds. Chem. Rev. 120, 7348-7398 (2020).

24 Brown, H. C. \& Rao, B. C. S. A new technique for the conversion of olefins into organoboranes and related alcohols. J. Am. Chem. Soc. 78, 5694-5695 (1956).

25 Matteson, D. S. \& Mah, R. W. H. Neighboring boron in nucleophilic displacement. J. Am. Chem. Soc. 85, 2599-2603 (1963).

26 Zweifel, G., Arzoumanian, H. \& Whitney, C. C. A convenient stereoselective synthesis of substituted alkenes via hydroboration-iodination of alkynes. J. Am. Chem. Soc. 89, 3652-3653 (1967).

27 Aggarwal, V. K., Fang, G. Y. \& Schmidt, A. T. Synthesis and applications of chiral organoboranes generated from sulfonium ylides. J. Am. Chem. Soc. 127, 1642-1643 (2005). 
28 Brown, H. C. \& Jadhav, P. K. Asymmetric carbon-carbon bond formation via Ballyldiisopinocampheylborane. Simple synthesis of secondary homoallylic alcohols with excellent enantiomeric purities. J. Am. Chem. Soc. 105, 2092-2093 (1983).

29 Miyaura, N., Ishiyama, T., Ishikawa, M. \& Suzuki, A. Palladium-catalyzed crosscoupling reactions of B-alkyl-9-BBN or trialkylboranes with aryl and 1-alkenyl halides. Tetrahedron Lett. 27, 6369-6372 (1986).

30 Molander, G. A. \& Ito, T. Cross-coupling reactions of potassium alkyltrifluoroborates with aryl and 1-alkenyl trifluoromethanesulfonates. Org. Lett. 3, 393-396 (2001).

31 Molander, G. A., Colombel, V. \& Braz, V. A. Direct alkylation of heteroaryls using potassium alkyl- and alkoxymethyltrifluoroborates. Org. Lett. 13, 1852-1855 (2011).

32 Yasu, Y., Koike, T. \& Akita, M. Visible light-induced selective generation of radicals from organoborates by photoredox catalysis. Adv. Synth. Catal. 354, 3414-3420 (2012).

33 Tellis, J. C., Primer, D. N. \& Molander, G. A. Single-electron transmetalation in organoboron cross-coupling by photoredox/nickel dual catalysis. Science 345, 433 (2014).

34 Duret, G., Quinlan, R., Bisseret, P. \& Blanchard, N. Boron chemistry in a new light. Chem. Sci. 6, 5366-5382 (2015).

35 Cazorla, C., Métay, E. \& Lemaire, M. Oxidative nucleophilic substitution: transformation of alkylboronic derivatives. Tetrahedron 67, 8615-8621 (2011).

36 Inagi, S. \& Fuchigami, T. Electrochemical properties and reactions of organoboron compounds. Curr. Opin. Electrochem. 2, 32-37 (2017).

37 Grayson, J. D., Dennis, F. M., Robertson, C. C. \& Partridge, B. M. Chan-Lam amination of secondary and tertiary benzylic boronic esters. J. Org. Chem. 86, 9883-9897 (2021).

38 Yan, M., Kawamata, Y. \& Baran, P. S. Synthetic organic electrochemical methods since 2000: on the verge of a renaissance. Chem. Rev. 117, 13230-13319 (2017).

39 Yan, M., Kawamata, Y. \& Baran, P. S. Synthetic organic electrochemistry: calling all engineers. Angew. Chem. Int. Ed. 57, 4149-4155 (2018).

40 Zhu, C., Ang, N. W. J., Meyer, T. H., Qiu, Y. \& Ackermann, L. Organic electrochemistry: molecular syntheses with potential. ACS Cent. Sci. 7, 415-431 (2021).

41 Sandford, C. et al. A synthetic chemist's guide to electroanalytical tools for studying reaction mechanisms. Chem. Sci. 10, 6404-6422 (2019).

42 Peters, B. K. et al. Scalable and safe synthetic organic electroreduction inspired by Liion battery chemistry. Science 363, 838 (2019).

$43 \mathrm{Hu}$, P. et al. Electroreductive olefin-ketone coupling. J. Am. Chem. Soc. 142, 2097920986 (2020).

44 Shin, S. J. et al. Thin-layer electroanalysis microchip reveals more about electrochemical reactions of organic compounds. Preprint at. doi:10.33774/chemrxiv-2021-kpk93 (2021).

45 Kotsinaris, A., Kyriacou, G. \& Lambrou, C. H. Electrochemical reduction of dichloromethane to higher hydrocarbons. J. Appl. Electrochem. 28, 613-616 (1998).

46 Chen, N., Ye, Z. \& Zhang, F. Recent progress on electrochemical synthesis involving carboxylic acids. Org. Biomol. Chem. 19, 5501-5520 (2021).

$47 \mathrm{Li}, \mathrm{Y}$. et al. Selectivity in the addition of electron-deficient radicals to the $C 2$ position of Indoles. Eur. J. Org. Chem. 2020, 5828-5832 (2020).

48 Taylor, A. P. et al. Modern advances in heterocyclic chemistry in drug discovery. Org. Biomol. Chem. 14, 6611-6637 (2016). 
49 Mayr, H. \& Minegishi, S. First direct observation of the two distinct steps in an $\mathrm{S}_{\mathrm{N}} 1$ Reaction. Angew. Chem. Int. Ed. 41, 4493-4495 (2002). 